\title{
STATION CORRECTION ANALYSIS FOR SURFACE-WAVE MAGNITUDES OF NEW ZEALAND EARTHQUAKES
}

\author{
D.A. Rhoades ${ }^{1,2}$ and D.J. Dowrick ${ }^{1,3}$
}

\begin{abstract}
SUMMARY
Station terms and standard errors are presented for 345 world-wide stations used in the determination of surface-wave magnitudes of 190 selected New Zealand earthquakes over the period 1901-1993 [1]. These will facilitate the estimation of surface-wave magnitudes of other earthquakes in the New Zealand region. The station terms and the residuals from the linear model used to estimate them are both found to be weakly related to the mean distance from the earthquakes recorded by each station. The horizontal and vertical components at a given site are treated as separate stations. The station term for the horizontal component tends to exceed that for the vertical component at mean distances in the $20^{\circ}-40^{\circ}$ range.
\end{abstract}

\section{INTRODUCTION}

This paper describes the station correction analysis for surfacewave magnitudes of a selection of 190 of the larger New Zealand earthquakes over the period 1901 to 1993 [1]. It follows a similar study $[2,3]$ carried out on a smaller selection of earthquakes with data from fewer stations. The methodology of the previous study has been followed in the main. An exception, discussed below, is in the treatment of the horizontal and vertical components at a single station.

The larger data set analysed in the present study allows station terms to be estimated for many more stations. It also offers the prospect of improved estimates of the station terms for the 96 stations included in the previous study.

Estimates of $M_{S}$ are normally made only from data recorded at distances less than $160^{\circ}$, e.g., as practised in the ISC Bulletin. This would exclude data from many stations in Europe, as seen in Figure 1, reducing our data set for New Zealand earthquakes from 2040 station observations of $M_{S}$ to 1577 . However, as found previously [2], data at distances $>160^{\circ}$ is made admissible by correcting for bias by calculating station corrections using analysis of variance.

\section{METHOD}

The data set used for this study has been described elsewhere [1], together with the computation of surface-wave magnitudes at each station from the seismograph records.

The estimation of station terms has been carried out simultaneously with the estimation of the surface-wave magnitudes, by fitting an analysis-of-variance model incorporating both earthquake terms and station terms. The model used is as follows [2]:

$$
M_{i j}=m_{i}+c_{j}+e_{i j}
$$

where $M_{i j}$ is the magnitude of the $i$ th earthquake computed from the seismographic record at the $j$ th station, $m_{i}$ is the "true" average magnitude of the $i$ th earthquake, $c_{j}$ is the fixed effect of the $j$ th station, and the $e_{i j}$ are independent normally distributed random errors with a common variance $\sigma^{2}$ that combines both observational inaccuracy and modelling deficiency. The modelling deficiency includes differences in the average attenuation along the paths to the stations, but as we are concerned solely with New Zealand events the paths to any given station will not vary greatly and the differences in average attenuation are therefore not expected to be large [2]. The above model is readily fitted by the method of ordinary least squares, although solving for such a large number of parameters (535) as are in the present study is demanding of computer memory. The size of the problem was substantially reduced by omitting from the data set, for model-fitting purposes, those stations which have only one observation. These stations contribute nothing to the estimation of magnitude, but their station terms can be estimated separately by $\hat{c}_{j}=M_{i j}-\hat{m}_{i}$, and their standard errors by $S\left(\hat{c}_{j}\right)=\left[S\left(\hat{m}_{i}\right)^{2}+s^{2}\right]^{1 / 2}$, where $S\left(\hat{m}_{i}\right)$ is the standard error of the estimated magnitude and $s$ is the residual standard deviation from model (1).

\footnotetext{
${ }^{1}$ Institute of Geological \& Nuclear Sciences, Lower Hutt

${ }^{2}$ Member

${ }^{3}$ Fellow
} 


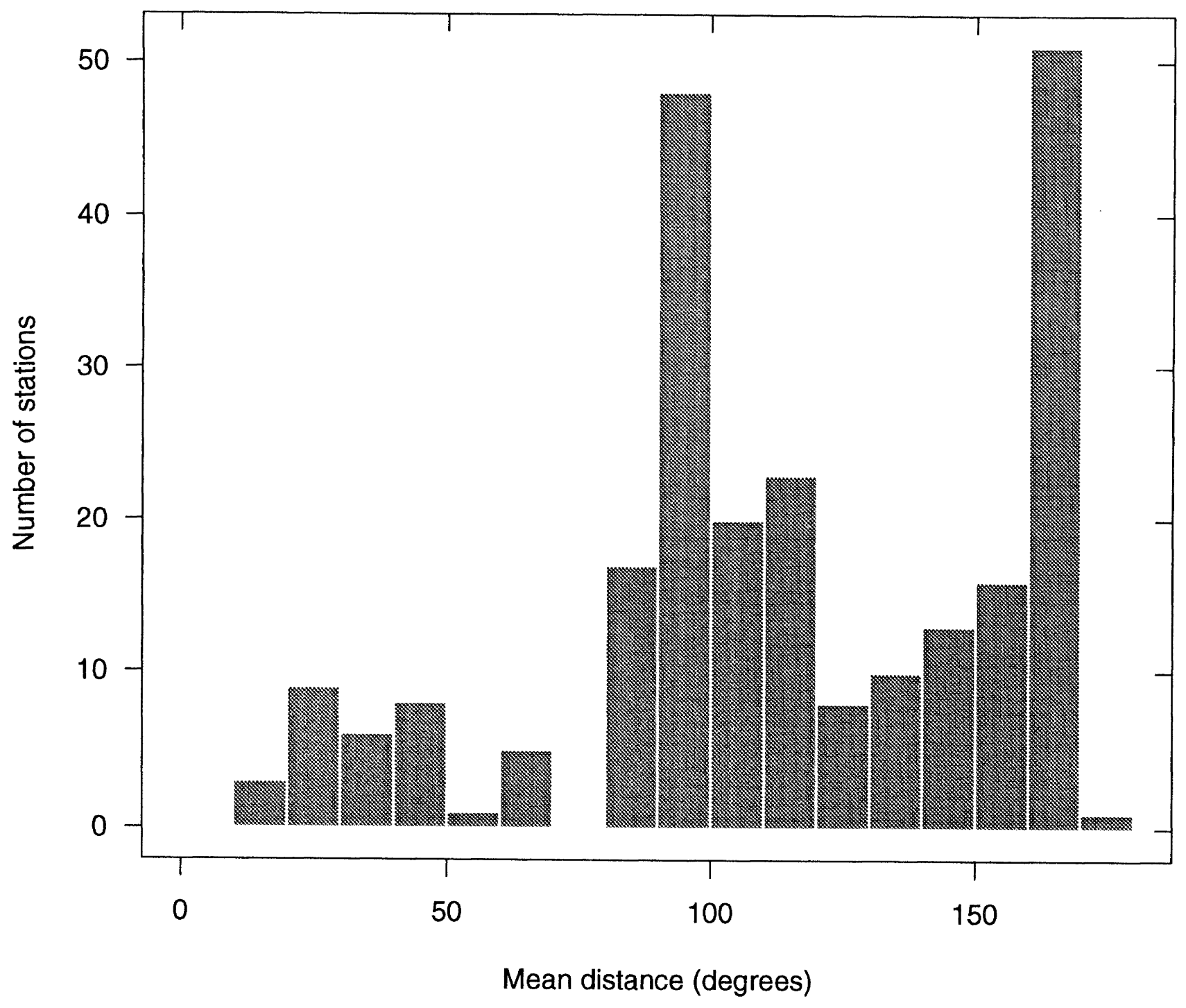

Figure 1 Histogram of mean distances from New Zealand earthquake sources of the stations outside of New Zealand used to determine surface-wave magnitudes.

\section{RESULTS}

The station terms, $\hat{c}$, and standard errors, $S(\hat{c})$, are listed in Table 1, together with the mean earthquake-station distance, $D$ (in degrees), and the number of observations, $N$, at each station. For stations included in the the previous study [2], the station terms do not differ greatly in most cases from the values given previously, but many of the standard errors are smaller because of the larger number of observations contributing to the estimates. The residual standard deviation from model (1) is given by $s=0.200$.

The three or four letter station codes are those used by the ISC $[4,5]$. In some cases a single letter code ("M" or "Z") follows the station code. Some earlier events were recorded on undamped Milne seismographs at certain stations. These observations have been treated as belonging to a different station from later recordings made at the same sites with damped seismographs, with an "M" added after the station code to distinguish the Milne instrument. Likewise horizontal and vertical components at the same site have been treated as separate stations in all cases, and a "Z" following the station code distinguishes the vertical component, where available. A somewhat different practice was adopted in the previous study [2], in which horizontal and vertical components were regarded as coming from the same station, and therefore averaged, except where they were found to differ significantly.

The linear model (1) does not allow independent estimation of all parameters; one parameter, or linear combination of parameters, must be fixed arbitrarily. As in the previous study [2], the station "UPP" (Uppsala) was chosen as a reference station and its station term arbitrarily set to zero, with a consequential systematic effect on all estimated magnitudes. Since the mean of the station terms turned out to be 0.001 , the results are essentially the same as if the mean of the station terms had been set to zero.

The station terms and standard errors may be used to streamline the computation of surface-wave magnitudes of New Zealand earthquakes in future studies, by correcting each station observation and averaging all corrected observations to produce 
Table 1: Station statistics and parameter estimates: Mean earthquake-station distance, $D$, in degrees, number of observations $N$, estimated station term $\hat{c}$, and standard error $S(\hat{c})$.

\begin{tabular}{|c|c|c|c|c|}
\hline Station & $D$ & $N$ & $\hat{c}$ & $S(\hat{c})$ \\
\hline AAS Z & 70 & 2 & 0.22 & 0.151 \\
\hline $\mathrm{ABE}$ & 164 & 4 & 0.12 & 0.111 \\
\hline $\mathrm{ADE} M$ & 24 & 1 & -0.56 & 0.259 \\
\hline $\mathrm{ADE}$ & 31 & 1 & -0.05 & 0.212 \\
\hline AFI & 27 & 14 & -0.27 & 0.071 \\
\hline AFI Z & 27 & 10 & -0.60 & 0.079 \\
\hline AGR & 113 & 1 & -0.14 & 0.211 \\
\hline AKU Z & 154 & 2 & 0.23 & 0.150 \\
\hline $\mathrm{ALG}$ & 173 & 7 & 0.19 & 0.087 \\
\hline ALQ & 101 & 2 & -0.06 & 0.151 \\
\hline ALQZ & 105 & 19 & 0.05 & 0.063 \\
\hline ANMO Z & 106 & 2 & 0.27 & 0.151 \\
\hline $\mathrm{ANN}$ & 150 & 1 & 0.47 & 0.207 \\
\hline ANN Z & 150 & 1 & 0.27 & 0.207 \\
\hline ANR & 122 & 1 & -0.18 & 0.208 \\
\hline ANR Z & 122 & 1 & -0.03 & 0.208 \\
\hline APP Z & 156 & 1 & 0.05 & 0.214 \\
\hline ARU & 136 & 2 & -0.12 & 0.149 \\
\hline ARU Z & 136 & 2 & -0.12 & 0.149 \\
\hline ASPA Z & 37 & 24 & 0.06 & 0.060 \\
\hline BAK & 140 & 11 & 0.43 & 0.080 \\
\hline BAK Z & 139 & 5 & 0.35 & 0.087 \\
\hline BCAO Z & 140 & 1 & -0.31 & 0.214 \\
\hline $\mathrm{BEO}$ & 159 & 12 & 0.09 & 0.069 \\
\hline BER & 158 & 2 & -0.04 & 0.152 \\
\hline BER Z & 161 & 1 & -0.24 & 0.209 \\
\hline BID M & 168 & 2 & 0.30 & 0.205 \\
\hline BJI & 96 & 16 & -0.27 & 0.065 \\
\hline BJI Z & 96 & 18 & -0.16 & 0.064 \\
\hline BKS & 96 & 6 & -0.09 & 0.093 \\
\hline BKS Z & 96 & 7 & 0.04 & 0.088 \\
\hline BLV & 125 & 1 & 0.17 & 0.243 \\
\hline BMO & 104 & 4 & -0.38 & 0.112 \\
\hline BMO Z & 105 & 5 & -0.15 & 0.104 \\
\hline BNS Z & 165 & 1 & -0.23 & 0.208 \\
\hline BOM M & 113 & 1 & 0.05 & 0.243 \\
\hline BOM & 111 & 23 & -0.07 & 0.056 \\
\hline BRA & 159 & 1 & -0.72 & 0.212 \\
\hline BRG & 162 & 10 & 0.19 & 0.077 \\
\hline BRG Z & 162 & 11 & 0.10 & 0.075 \\
\hline BRK Z & 96 & 1 & 0.21 & 0.207 \\
\hline BRS Z & 21 & 2 & 0.33 & 0.150 \\
\hline BRU & 168 & 12 & 0.25 & 0.069 \\
\hline BRU Z & 168 & 2 & 0.18 & 0.150 \\
\hline BTO & 100 & 9 & -0.21 & 0.079 \\
\hline BTO Z & 100 & 2 & -0.39 & 0.149 \\
\hline BUD & 162 & 10 & 0.12 & 0.074 \\
\hline BUL & 116 & 1 & -0.28 & 0.208 \\
\hline BUL Z & 116 & 2 & 0.13 & 0.150 \\
\hline CAL M & 10 & 1 & 0.00 & 0.243 \\
\hline $\mathrm{CAL}$ & 103 & 1 & -0.03 & 0.211 \\
\hline CAP M & 10 & 1 & 0.61 & 0.269 \\
\hline CAR & 120 & 2 & -0.07 & 0.150 \\
\hline CAR Z & 120 & 2 & 0.03 & 0.150 \\
\hline CBD & 133 & 1 & -0.17 & 0.229 \\
\hline CBM Z & 13 & 1 & -0.35 & 0.209 \\
\hline
\end{tabular}

\begin{tabular}{|c|c|c|c|c|}
\hline Station & $D$ & $N$ & $\hat{c}$ & $S(\hat{c})$ \\
\hline $\mathrm{CDU}$ & 96 & 2 & -0.29 & 0.151 \\
\hline $\mathrm{CD} 2$ & 96 & 6 & -0.15 & 0.093 \\
\hline $\mathrm{CD} 2 \mathrm{Z}$ & 96 & 8 & -0.18 & 0.083 \\
\hline $\mathrm{CEH} \mathrm{Z}$ & 126 & 3 & -0.19 & 0.126 \\
\hline CFF & 167 & 1 & -0.55 & 0.211 \\
\hline $\mathrm{CHG} \mathrm{Z}$ & 92 & 1 & 0.59 & 0.225 \\
\hline CLL Z & 162 & 8 & 0.05 & 0.084 \\
\hline $\mathrm{CMB}$ & 97 & 1 & 0.15 & 0.209 \\
\hline $\mathrm{CNH}$ & 94 & 2 & -0.16 & 0.150 \\
\hline $\mathrm{CN} 2$ & 95 & 12 & -0.26 & 0.072 \\
\hline $\mathrm{CN} 2 \mathrm{Z}$ & 95 & 15 & -0.10 & 0.068 \\
\hline $\mathrm{COL} \mathrm{Z}$ & 105 & 1 & 0.03 & 0.210 \\
\hline COP Z & 160 & 6 & 0.16 & 0.093 \\
\hline COR M & 8 & 1 & 0.37 & 0.269 \\
\hline $\mathrm{CPO}$ & 119 & 3 & 0.00 & 0.130 \\
\hline $\mathrm{CPO} Z$ & 116 & 4 & -0.09 & 0.115 \\
\hline CTA & 31 & 1 & -0.36 & 0.213 \\
\hline CTA Z & 32 & 9 & -0.09 & 0.085 \\
\hline CUL M & 10 & 1 & 0.62 & 0.269 \\
\hline $\mathrm{DAG}$ & 143 & 1 & 0.20 & 0.207 \\
\hline $\mathrm{DAG} Z$ & 145 & 3 & 0.07 & 0.127 \\
\hline $\mathrm{DBN}$ & 166 & 28 & 0.42 & 0.053 \\
\hline DBN Z & 16 & 18 & 0.21 & 0.058 \\
\hline DJA & 64 & 1 & -0.12 & 0.222 \\
\hline DL2 & 93 & 4 & -0.24 & 0.110 \\
\hline DL2 Z & 92 & 8 & -0.38 & 0.083 \\
\hline DOU Z & 16 & 5 & 0.15 & 0.101 \\
\hline DUG Z & 10 & 2 & -0.12 & 0.149 \\
\hline DUR & 167 & 1 & -0.08 & 0.212 \\
\hline EDI M & 166 & 2 & -0.02 & 0.205 \\
\hline EGE & 164 & 12 & -0.01 & 0.071 \\
\hline ERE & 141 & 1 & 0.11 & 0.208 \\
\hline ERE Z & 144 & 1 & 0.27 & 0.207 \\
\hline FUR & 165 & 2 & 0.28 & 0.151 \\
\hline FUR Z & 164 & 3 & 0.10 & 0.124 \\
\hline FVM Z & 116 & 2 & 0.39 & 0.152 \\
\hline GDH Z & 138 & 1 & -0.19 & 0.213 \\
\hline GLD Z & 111 & 6 & 0.10 & 0.093 \\
\hline GOGA Z & 117 & 1 & 0.45 & 0.209 \\
\hline GOL Z & 112 & 8 & 0.03 & 0.083 \\
\hline GRA & 165 & 1 & 0.15 & 0.213 \\
\hline GRE Z & 165 & 1 & -0.13 & 0.214 \\
\hline GRF Z & 165 & 21 & 0.08 & 0.061 \\
\hline GRM Z & 94 & 1 & -0.02 & 0.207 \\
\hline GRO & 144 & 1 & 0.07 & 0.207 \\
\hline GRO Z & 144 & 1 & 0.47 & 0.207 \\
\hline GTA & 104 & 6 & -0.33 & 0.093 \\
\hline GTA Z & 104 & 8 & -0.14 & 0.083 \\
\hline GTT & 165 & 9 & 0.11 & 0.079 \\
\hline GTT Z & 165 & 4 & 0.23 & 0.099 \\
\hline GUA Z & 60 & 2 & -0.42 & 0.149 \\
\hline GUMO Z & 61 & 6 & -0.23 & 0.095 \\
\hline GYA & 91 & 10 & -0.29 & 0.076 \\
\hline GYA Z & 92 & 10 & -0.30 & 0.077 \\
\hline GZH & 84 & 6 & -0.30 & 0.093 \\
\hline GZH Z & 85 & 6 & -0.43 & 0.093 \\
\hline
\end{tabular}




\begin{tabular}{|c|c|c|c|c|}
\hline Station & $D$ & $N$ & $\hat{c}$ & $S(\hat{c})$ \\
\hline HAM & 164 & 9 & 0.38 & 0.079 \\
\hline HAM Z & 164 & 4 & 0.24 & 0.110 \\
\hline HAU Z & 167 & 1 & -0.23 & 0.210 \\
\hline HEL & 151 & 1 & -0.23 & 0.211 \\
\hline HFS Z & 156 & 5 & -0.05 & 0.105 \\
\hline $\mathrm{HHC}$ & 99 & 7 & -0.22 & 0.088 \\
\hline HHC Z & 99 & 9 & -0.11 & 0.080 \\
\hline HLL & 32 & 5 & 0.08 & 0.118 \\
\hline HLL Z & 32 & 5 & 0.01 & 0.118 \\
\hline HLW & 145 & 4 & 0.42 & 0.100 \\
\hline HOF Z & 163 & 1 & -0.04 & 0.208 \\
\hline HON Z & 67 & 7 & -0.39 & 0.088 \\
\hline HRV & 139 & 1 & -0.22 & 0.207 \\
\hline HRV Z & 132 & 2 & -0.12 & 0.152 \\
\hline HUR & 160 & 2 & -0.12 & 0.151 \\
\hline HYD & 105 & 12 & 0.09 & 0.070 \\
\hline IRK & 111 & 11 & -0.37 & 0.072 \\
\hline IRK Z & 111 & 5 & -0.26 & 0.099 \\
\hline ISA Z & 96 & 1 & 0.15 & 0.209 \\
\hline JCT Z & 104 & 5 & 0.13 & 0.101 \\
\hline JEN & 164 & 11 & -0.02 & 0.071 \\
\hline JEN Z & 164 & 9 & 0.03 & 0.081 \\
\hline JEN 2 & 164 & 9 & -0.10 & 0.077 \\
\hline KAR & 167 & 1 & 0.04 & 0.208 \\
\hline KAT & 134 & 2 & -0.08 & 0.149 \\
\hline KAT Z & 135 & 1 & 0.27 & 0.207 \\
\hline KER & 69 & 1 & -0.62 & 0.211 \\
\hline KEV Z & 148 & 1 & -0.31 & 0.210 \\
\hline KEW M & 138 & 2 & 0.06 & 0.205 \\
\hline KEW & 168 & 6 & 0.34 & 0.093 \\
\hline KEW Z & 166 & 3 & 0.28 & 0.125 \\
\hline $\mathrm{KHC}$ & 164 & 14 & 0.04 & 0.069 \\
\hline $\mathrm{KHCZ}$ & 164 & 15 & 0.11 & 0.067 \\
\hline KIR & 149 & 13 & 0.04 & 0.068 \\
\hline KIR Z & 150 & 14 & 0.10 & 0.066 \\
\hline KIS & 153 & 1 & 0.06 & 0.208 \\
\hline KJF Z & 149 & 2 & 0.13 & 0.150 \\
\hline KMI & 93 & 14 & -0.13 & 0.068 \\
\hline KMI Z & 93 & 14 & -0.01 & 0.069 \\
\hline KOD M & 101 & 1 & 0.30 & 0.269 \\
\hline KOD & 101 & 7 & 0.11 & 0.086 \\
\hline KRA & 159 & 6 & 0.23 & 0.093 \\
\hline KRA Z & 159 & 12 & 0.20 & 0.072 \\
\hline $\mathrm{KSH}$ & 118 & 6 & -0.05 & 0.093 \\
\hline KSH Z & 119 & 5 & -0.26 & 0.100 \\
\hline KSN & 156 & 1 & 0.12 & 0.229 \\
\hline KUC & 149 & 6 & 0.04 & 0.093 \\
\hline KUR & 89 & 2 & -0.04 & 0.149 \\
\hline KUR Z & 89 & 2 & -0.19 & 0.149 \\
\hline LEI & 163 & 10 & 0.11 & 0.074 \\
\hline LEI Z & 162 & 3 & 0.14 & 0.125 \\
\hline LEM & 158 & 2 & -0.02 & 0.150 \\
\hline LEN Z & 150 & 1 & 0.05 & 0.214 \\
\hline LIC Z & 145 & 12 & 0.03 & 0.075 \\
\hline LJU & 164 & 3 & 0.15 & 0.124 \\
\hline LOR Z & 170 & 2 & 0.24 & 0.152 \\
\hline LPA & 90 & 6 & -0.16 & 0.093 \\
\hline LPA Z & 91 & 16 & -0.02 & 0.065 \\
\hline
\end{tabular}

\begin{tabular}{|c|c|c|c|c|}
\hline Station & $D$ & $N$ & $\hat{c}$ & $S(\hat{c})$ \\
\hline LPB & 99 & 23 & -0.06 & 0.057 \\
\hline LPB Z & 99 & 28 & -0.08 & 0.055 \\
\hline LSA & 101 & 1 & -0.12 & 0.207 \\
\hline LSA Z & 101 & 1 & 0.08 & 0.207 \\
\hline LZH & 100 & 14 & -0.21 & 0.069 \\
\hline LZH Z & 100 & 10 & -0.29 & 0.078 \\
\hline MAG & 103 & 1 & 0.27 & 0.208 \\
\hline MAK & 145 & 2 & 0.00 & 0.152 \\
\hline MAK Z & 150 & 1 & 0.03 & 0.214 \\
\hline MAT & 82 & 4 & -0.36 & 0.111 \\
\hline MAT Z & 84 & 20 & -0.37 & 0.061 \\
\hline MAW Z & 63 & 1 & -0.23 & 0.207 \\
\hline MBO & 146 & 2 & 0.66 & 0.152 \\
\hline MBO Z & 147 & 3 & 0.52 & 0.126 \\
\hline MCWV Z & 127 & 3 & 0.06 & 0.125 \\
\hline MDJ & 94 & 5 & -0.17 & 0.100 \\
\hline MDJ Z & 94 & 7 & -0.16 & 0.088 \\
\hline MEL M & 24 & 7 & 0.15 & 0.096 \\
\hline MEL & 23 & 20 & -0.04 & 0.065 \\
\hline MHC & 96 & 2 & -0.23 & 0.149 \\
\hline MHC Z & 96 & 2 & -0.23 & 0.149 \\
\hline MIAR Z & 116 & 2 & -0.01 & 0.150 \\
\hline MIR & 48 & 1 & 0.43 & 0.208 \\
\hline MIR Z & 48 & 1 & 0.45 & 0.208 \\
\hline MOS & 149 & 4 & -0.01 & 0.109 \\
\hline MOS Z & 148 & 1 & -0.03 & 0.207 \\
\hline MOX & 164 & 17 & 0.06 & 0.063 \\
\hline MOX Z & 164 & 18 & 0.07 & 0.062 \\
\hline MTA & 144 & 1 & 0.77 & 0.207 \\
\hline MTA Z & 144 & 1 & 0.87 & 0.207 \\
\hline MUN & 49 & 3 & -0.06 & 0.125 \\
\hline MUN Z & 48 & 9 & -0.17 & 0.080 \\
\hline NAI Z & 126 & 1 & -0.03 & 0.207 \\
\hline NDI & 116 & 1 & 0.06 & 0.212 \\
\hline NDI Z & 116 & 1 & -0.06 & 0.212 \\
\hline NEW Z & 106 & 4 & 0.36 & 0.110 \\
\hline NJI & 88 & 1 & 0.08 & 0.209 \\
\hline NJ2 & 89 & 5 & -0.32 & 0.100 \\
\hline NJ2 Z & 89 & 6 & -0.49 & 0.094 \\
\hline NNA Z & 97 & 5 & -0.41 & 0.100 \\
\hline NOU & 18 & 1 & 0.28 & 0.213 \\
\hline NUR Z & 152 & 9 & 0.00 & 0.084 \\
\hline NVL & 68 & 4 & -0.01 & 0.110 \\
\hline NVL Z & 69 & 4 & -0.01 & 0.110 \\
\hline NWAO & 46 & 8 & -0.39 & 0.088 \\
\hline NWAO Z & 45 & 12 & -0.41 & 0.075 \\
\hline OBN & 149 & 3 & -0.02 & 0.124 \\
\hline OBN Z & 149 & 5 & 0.18 & 0.105 \\
\hline OSA & 83 & 5 & 0.03 & 0.101 \\
\hline PAR & 170 & 19 & 0.31 & 0.060 \\
\hline PAS Z & 94 & 1 & 0.32 & 0.208 \\
\hline PER M & 48 & 2 & 0.00 & 0.205 \\
\hline PER & 46 & 3 & 0.37 & 0.127 \\
\hline PET Z & 96 & 1 & 0.69 & 0.208 \\
\hline PMG Z & 39 & 1 & -0.07 & 0.214 \\
\hline PMR & 105 & 2 & -0.14 & 0.150 \\
\hline PMR Z & 106 & 6 & -0.16 & 0.093 \\
\hline POL & 167 & 3 & 0.08 & 0.129 \\
\hline
\end{tabular}




\begin{tabular}{|c|c|c|c|c|}
\hline Station & $D$ & $N$ & $\hat{c}$ & $\hat{S(\hat{c})}$ \\
\hline $\mathrm{POO}$ & 113 & 1 & 0.41 & 0.210 \\
\hline POT & 164 & 3 & 0.24 & 0.131 \\
\hline PPT Z & 45 & $\begin{array}{l}3 \\
2\end{array}$ & -0.05 & 0.149 \\
\hline PRA & 163 & $\begin{array}{r}2 \\
18\end{array}$ & 0.03 & 0.060 \\
\hline PRA Z & 163 & $\begin{array}{l}18 \\
12\end{array}$ & 0.09 & 0.071 \\
\hline PRU & 162 & 12 & 0.06 & 0.059 \\
\hline PRU Z & 163 & $\begin{array}{l}22 \\
25\end{array}$ & 0.13 & 0.059 \\
\hline PUL & 151 & 20 & 0.01 & 0.075 \\
\hline PUL Z & 151 & $\begin{array}{r}11 \\
8\end{array}$ & 0.08 & 0.082 \\
\hline QIZ & 84 & $\begin{array}{l}8 \\
6\end{array}$ & -0.26 & 0.093 \\
\hline QZH & 83 & $\begin{array}{l}0 \\
8\end{array}$ & -0.26 & 0.083 \\
\hline QZH Z & 83 & $\begin{array}{l}8 \\
7\end{array}$ & -0.33 & 0.087 \\
\hline RAC Z & 158 & 1 & -0.12 & 0.207 \\
\hline RAR & 26 & $\begin{array}{r}1 \\
13\end{array}$ & 0.01 & 0.072 \\
\hline RAR Z & 28 & $\begin{array}{l}13 \\
14\end{array}$ & -0.44 & 0.071 \\
\hline RIV & 20 & $\begin{array}{r}14 \\
125\end{array}$ & -0.06 & 0.042 \\
\hline RIV Z & 20 & $\begin{array}{r}125 \\
92\end{array}$ & -0.29 & 0.043 \\
\hline RJF Z & 173 & 92 & 0.04 & 0.212 \\
\hline ROX & 8 & 1 & 0.48 & 0.227 \\
\hline ROX Z & 8 & 1 & 0.33 & 0.227 \\
\hline RSCP Z & 122 & $\frac{1}{6}$ & -0.06 & 0.093 \\
\hline RSNY Z & 131 & $\begin{array}{l}0 \\
9\end{array}$ & 0.09 & 0.080 \\
\hline RSON Z & 124 & $\begin{array}{l}9 \\
7\end{array}$ & 0.03 & 0.088 \\
\hline RSSD Z & 116 & 3 & 0.02 & 0.125 \\
\hline SAO Z & 100 & $\begin{array}{l}3 \\
2\end{array}$ & $-0.06-$ & 0.150 \\
\hline SBA & 38 & $\begin{array}{r}2 \\
36\end{array}$ & 0.04 & 0.055 \\
\hline SBA Z & 38 & $\begin{array}{l}36 \\
34\end{array}$ & -0.23 & 0.056 \\
\hline SDN Z & 101 & $\begin{array}{r}34 \\
3\end{array}$ & -0.10 & 0.124 \\
\hline SEM & 122 & 3 & -0.02 & 0.208 \\
\hline SEM Z & 122 & 1 & 0.02 & 0.208 \\
\hline SHE & 141 & 1 & -0.13 & 0.207 \\
\hline SHE Z & 141 & 1 & 0.57 & 0.207 \\
\hline SHI M & 170 & $\frac{1}{2}$ & 0.34 & 0.205 \\
\hline SIM & 150 & 2 & 0.15 & 0.208 \\
\hline SIM Z & 150 & 1 & 0.22 & 0.208 \\
\hline SIT Z & 108 & 1 & 0.07 & 0.087 \\
\hline SJG & 122 & 7 & 0.12 & 0.208 \\
\hline SJG Z & 122 & 1 & -0.15 & 0.101 \\
\hline SKA & 157 & $\begin{array}{l}5 \\
1\end{array}$ & -0.54 & 0.212 \\
\hline SKO & 159 & $\begin{array}{l}1 \\
8\end{array}$ & 0.17 & 0.084 \\
\hline SKO Z & 159 & 8 & 0.05 & 0.074 \\
\hline SLL Z & 156 & 11 & 0.18 & 0.150 \\
\hline SLM Z & 119 & $\begin{array}{l}2 \\
5\end{array}$ & 0.01 & 0.101 \\
\hline SLR Z & 106 & 5 & 0.32 & 0.150 \\
\hline SMY Z & 94 & $\begin{array}{l}2 \\
6\end{array}$ & 0.02 & 0.093 \\
\hline SNY & 95 & $\begin{array}{l}6 \\
6\end{array}$ & -0.27 & 0.093 \\
\hline SNY Z & 94 & $\begin{array}{l}6 \\
9\end{array}$ & -0.23 & 0.080 \\
\hline SOC & 148 & 9 & 0.27 & 0.207 \\
\hline SOC Z & 148 & 1 & 0.17 & 0.207 \\
\hline SPA & 53 & 1 & -0.07 & 0.209 \\
\hline SPA Z & 50 & 26 & -0.14 & 0.058 \\
\hline SRO & 161 & $\begin{array}{r}20 \\
3\end{array}$ & 0.07 & 0.125 \\
\hline SSE & 87 & 10 & -0.18 & 0.063 \\
\hline SSE Z & 87 & 19 & -0.17 & 0.059 \\
\hline SSF & 86 & 25 & -0.21 & 0.213 \\
\hline SSH & 86 & 1 & 0.11 & 0.209 \\
\hline SSPA Z & 133 & 1 & 0.08 & 0.207 \\
\hline STR & 167 & 1 & 0.26 & 0.067 \\
\hline STR Z & 167 & $\begin{array}{r}14 \\
8\end{array}$ & 0.19 & 0.077 \\
\hline
\end{tabular}

\begin{tabular}{|c|c|c|c|c|}
\hline Station & $D$ & $N$ & $\hat{c}$ & $S(\hat{c})$ \\
\hline STU & 166 & 5 & 0.16 & 0.099 \\
\hline STU Z & 166 & 5 & 0.25 & 0.099 \\
\hline SUV M & 23 & 5 & 0.14 & 0.106 \\
\hline SUV & 24 & 11 & 0.15 & 0.078 \\
\hline SVE & 136 & 16 & -0.05 & 0.067 \\
\hline SVE Z & 136 & 7 & -0.13 & 0.082 \\
\hline SYD M & 20 & 3 & 0.12 & 0.144 \\
\hline TAS & 127 & 18 & -0.17 & 0.063 \\
\hline TAS Z & 127 & 3 & -0.68 & 0.125 \\
\hline TFO & 101 & 1 & 0.41 & 0.232 \\
\hline TFO Z & 100 & 4 & -0.20 & 0.115 \\
\hline TIA & 93 & 11 & -0.25 & 0.074 \\
\hline TIA Z & 93 & 10 & -0.20 & 0.077 \\
\hline TIK & 118 & 1 & 0.37 & 0.208 \\
\hline TIY & 96 & 9 & -0.19 & 0.080 \\
\hline TIY Z & 96 & 9 & -0.12 & 0.080 \\
\hline TLG & 120 & 1 & 0.19 & 0.208 \\
\hline TLG Z & 120 & 1 & 0.28 & 0.208 \\
\hline TNT M & 127 & 2 & -0.52 & 0.205 \\
\hline TPNV Z & 98 & 2 & 0.10 & 0.150 \\
\hline TRI & 166 & 3 & -0.04 & 0.131 \\
\hline TUC & 96 & 1 & 0.26 & 0.209 \\
\hline TUC Z & 97 & 3 & -0.23 & 0.128 \\
\hline TUL Z & 115 & 3 & 0.29 & 0.124 \\
\hline UBO & 107 & 3 & -0.06 & 0.134 \\
\hline UBO Z & 107 & 4 & -0.09 & 0.118 \\
\hline UPA & 114 & 1 & 0.13 & 0.209 \\
\hline UPA Z & 111 & 3 & -0.03 & 0.125 \\
\hline UPP & 156 & 35 & 0.00 & 0.000 \\
\hline UPP Z & 156 & 15 & 0.10 & 0.064 \\
\hline $\mathrm{UZH}$ & 158 & 1 & 0.06 & 0.208 \\
\hline VIC M & 103 & 1 & 0.57 & 0.243 \\
\hline VKA & 162 & 2 & -0.07 & 0.150 \\
\hline VKA Z & 161 & 6 & -0.03 & 0.093 \\
\hline VLA & 92 & 6 & -0.39 & 0.091 \\
\hline VLA Z & 92 & 4 & -0.42 & 0.109 \\
\hline WAR & 157 & 1 & -0.33 & 0.212 \\
\hline WAR Z & 157 & 1 & -0.11 & 0.212 \\
\hline WDC Z & 97 & 1 & 0.15 & 0.209 \\
\hline WET Z & 164 & 1 & -0.46 & 0.212 \\
\hline WHN & 90 & 10 & -0.24 & 0.076 \\
\hline WHN Z & 90 & 10 & -0.28 & 0.077 \\
\hline WIE & 164 & 6 & 0.08 & 0.091 \\
\hline WIE Z & 163 & 4 & 0.00 & 0.109 \\
\hline WIN Z & 119 & 2 & 0.24 & 0.152 \\
\hline WMOK Z & 111 & 2 & 0.28 & 0.149 \\
\hline WMO & 111 & 3 & -0.08 & 0.131 \\
\hline WMO Z & 110 & 6 & 0.15 & 0.097 \\
\hline WMQ & 114 & 3 & -0.23 & 0.125 \\
\hline WMQ Z & 114 & 7 & -0.27 & 0.088 \\
\hline XAN & 96 & 11 & -0.28 & 0.074 \\
\hline XAN Z & 95 & 3 & -0.27 & 0.124 \\
\hline YAK & 107 & 1 & 0.17 & 0.207 \\
\hline YAK Z & 107 & 1 & 0.17 & 0.207 \\
\hline YSS & 94 & 2 & -0.10 & 0.149 \\
\hline YSS Z & 94 & 2 & -0.17 & 0.149 \\
\hline ZOBO Z & 99 & 9 & -0.32 & 0.082 \\
\hline ZST Z & 159 & 1 & -0.34 & 0.207 \\
\hline
\end{tabular}


a best estimate. Thus, if some earthquake not in the present data set [1] has observed magnitudes $M_{j}$ at stations $j=1, \ldots, n$, then the surface-wave magnitude may be estimated by

$$
\hat{m}=\sum_{j=1}^{n}\left(M_{j}-\hat{c}_{j}\right) / n
$$

where $\hat{c}_{j}$ is the station term obtained from Table 1 . This estimate has standard error $S(\hat{m})$ given by

$S(\hat{m})=\left[n s^{2}+\sum_{j=1}^{n} S\left(\hat{c_{j}}\right)^{2}\right]^{1 / 2} / n$
Clearly, the greater the number of stations used, the smaller the standard error $S(\hat{m})$ will tend to be. The data in Table 1 allow an examination of possible relations between station effects and distance. In Figure 2 the station term is plotted against the mean distance of the station from earthquakes it recorded. Also plotted is a robust smooth trend line computed using the Splus function "lowess" [6]. It can be seen that the station term varies systematically with distance, being most negative $(-0.07)$ at about $90^{\circ}$ and most positive $(+0.18)$ at about $173^{\circ}$ (the greatest mean distance in the data set).

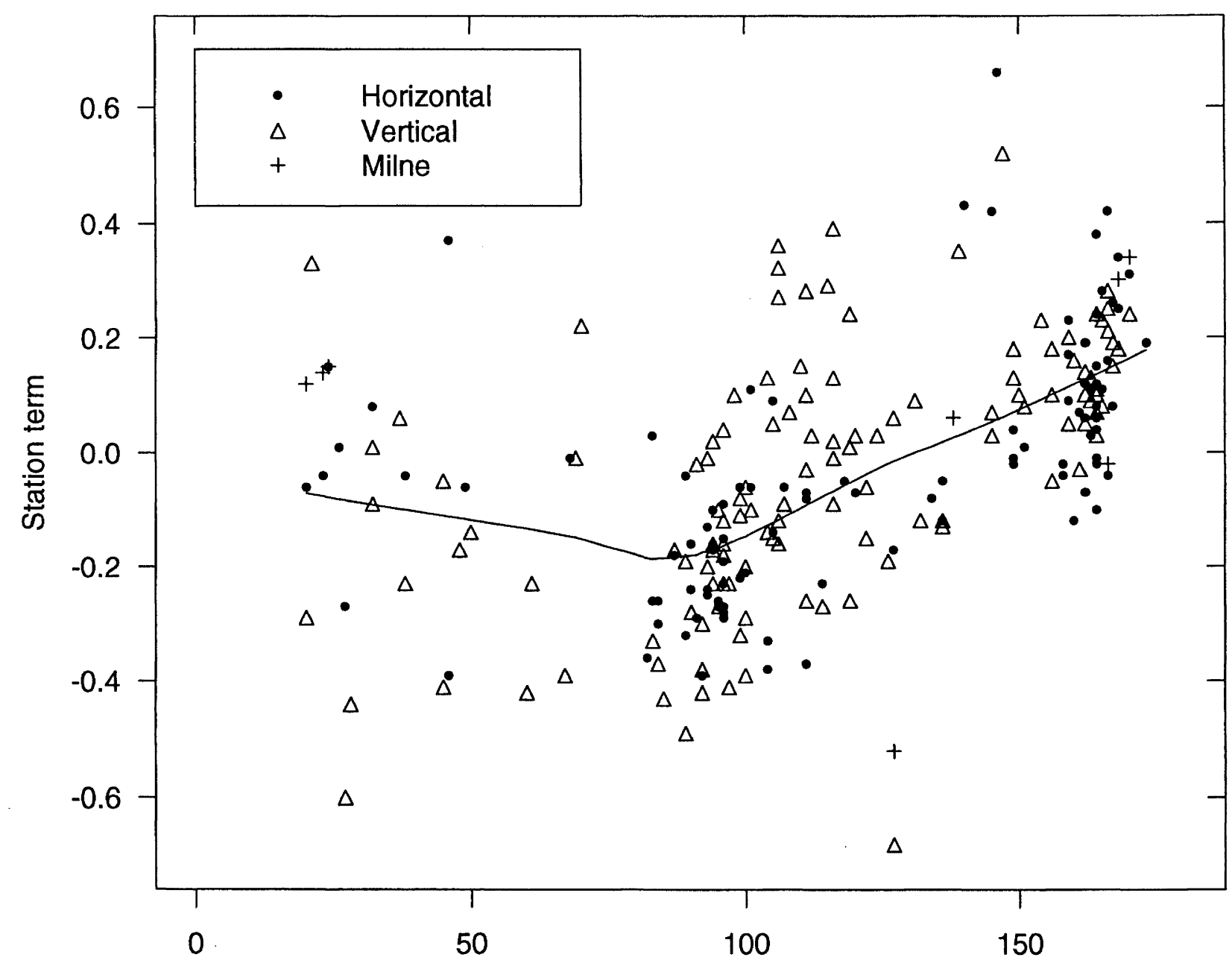

Mean distance from earthquake (degrees)

Figure 2 Plot of station terms against mean distance, in degrees, of New Zealand earthquakes from the station, distinguishing horizontal and vertical components (damped seismographs) and undamped Milne seismographs. A robust smooth trend, computed using the Splus "lowess" function [6], has been fitted through all the data. 
The trend in Figure 2 is consistent with the expected secondorder effects of the geometry of the world's surface on surfacewave attenuation. Surface-wave trains diverge out to distances of $90^{\circ}$ and then converge between $90^{\circ}$ and $180^{\circ}$. Hence the attenuation of surface waves will tend to be greater out to $90^{\circ}$ and less between $90^{\circ}$ and $180^{\circ}$, relative to the mean attenuation relation used for estimates of surface-wave magnitude at individual stations [1]. It should be no surprise, then, to see the decreasing trend in station terms out to $90^{\circ}$ and the increasing trend from $90^{\circ}$ to $180^{\circ}$.

The relatively small spread of station terms at mean distances greater than $150^{\circ}$ suggests less variability in this range. This is confirmed by a plot of the absolute value of the residuals from model (1) against distance (Figure 3), again with an accompanying trend line. Here, the residual $r_{i j}$ of the $i$ th earthquake at the $j$ th station is defined by

$$
r_{i j}=M_{i j}-\hat{m}_{j}-\hat{c}_{j}
$$

Figure 3 shows that the size of the residuals, although quite variable, decreases, on average, from about 0.13 to about 0.1 , as the distance increases from about $90^{\circ}$ to $173^{\circ}$. Thus, it is the stations at distances greater than $160^{\circ}$ which contribute data of highest precision to the estimation of New Zealand surfacewave magnitudes, provided that station corrections are made. This is a significant result, since it is common practice to use only stations at distances in the range $20^{\circ} \leq D \leq 160^{\circ}$ to estimate surface-wave magnitudes (e.g. the ISC Bulletin). The data at distances greater than $160^{\circ}$ are particularly important for New Zealand earthquakes because they comprise about a quarter of the total available data. Their relatively high precision makes them even more important.

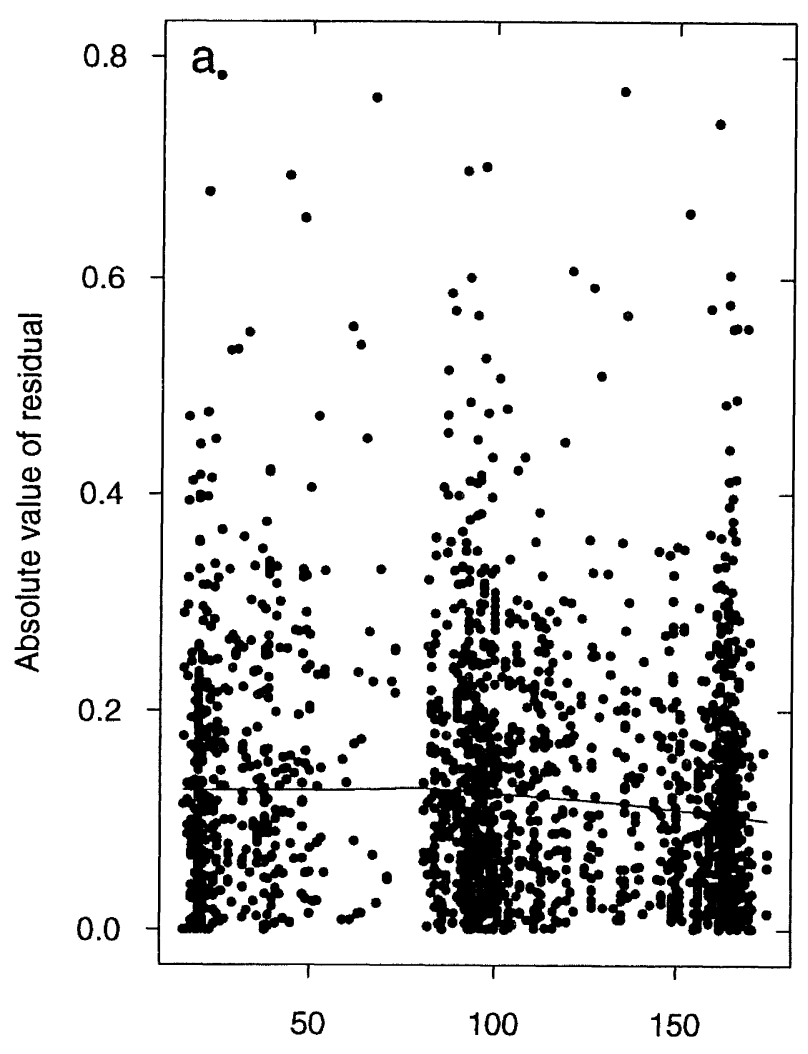

Earthquake-station distance (degrees)

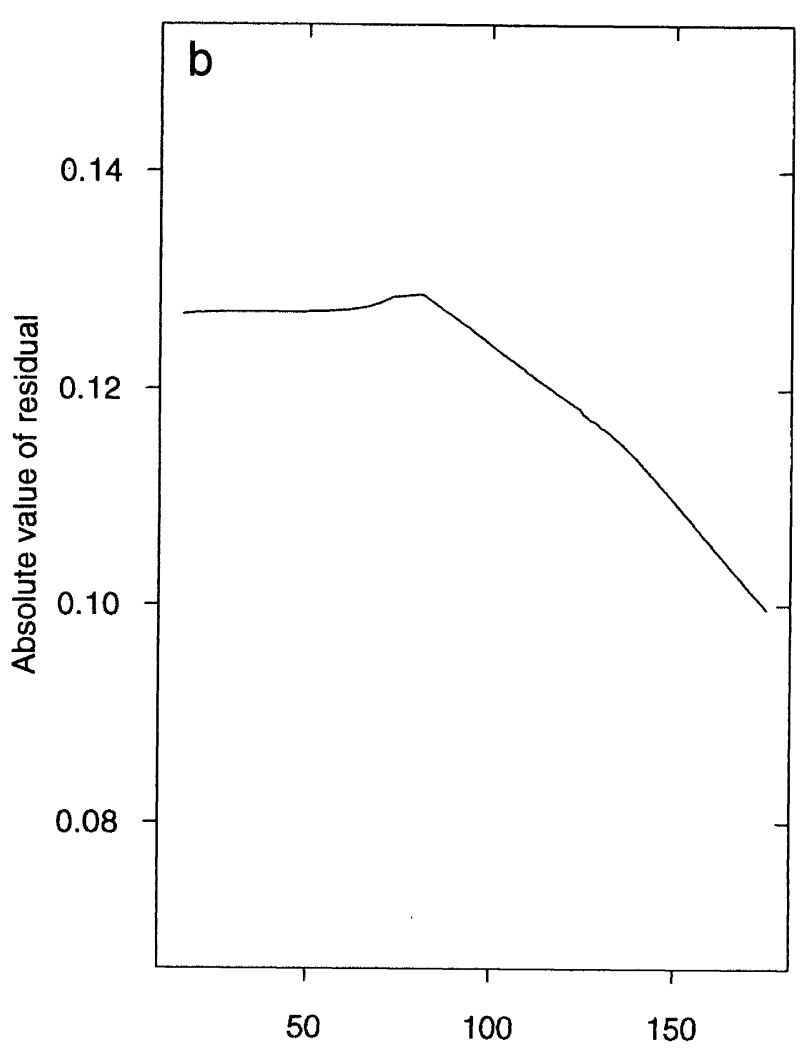

Earthquake-station distance (degrees)

Figure 3 (a) Plot of absolute value of residuals from model (1) of station observations against distance, in degrees, from earthquakes to station. Also shown is a robust smooth trend computed using Splus "lowess" function [6]. (b) Trend from (a) on enlarged scale. 


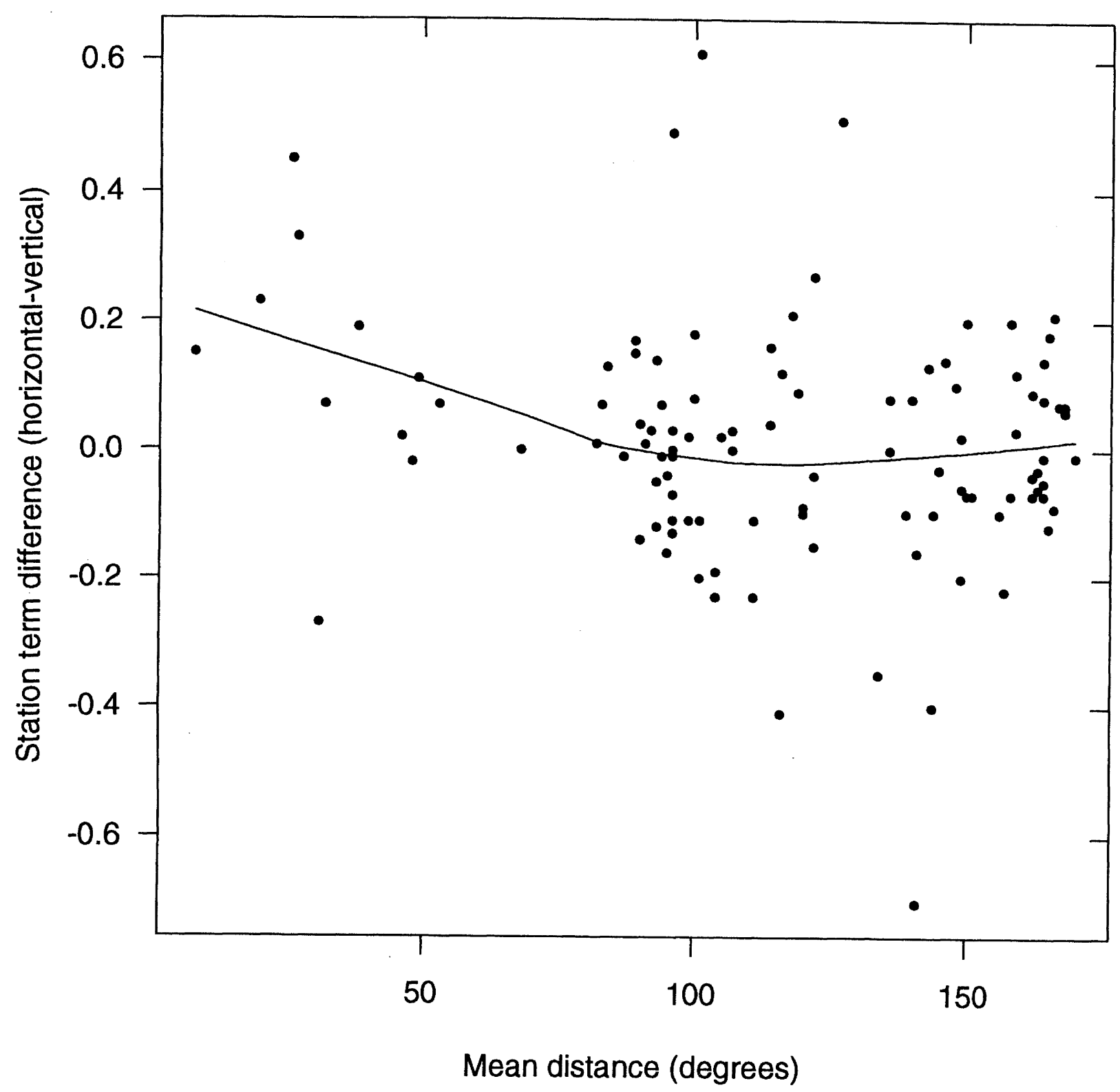

Figure 4 Difference of station terms estimated from the horizontal and vertical components recorded at a single site plotted against mean distance, in degrees, of New Zealand earthquakes from the site. A robust smooth trend, computed using the Splus "lowess" function [6], has been fitted through the data.

Although we have no data from stations at, or close to, a distance of $180^{\circ}$, it is noted that near the antipode the maximum phases of surface wave trains can arrive simultaneously from different directions. For large earthquakes, this effect may occur within about $4^{\circ}$ of the antipode, i.e. at distances $>176^{\circ}$.

Enhancements of peak amplitudes by superposition are clearly possible in this situation, and focusing with strong amplifications of up to an order of magnitude, within $2^{\circ}$ of the antipode, have been demonstrated [7] for body waves from the 1968 Inangahua, New Zealand, earthquake recorded in Spain. A surface-wave amplitude amplification by a factor of ten implies an overestimate of $M_{S}$ by an increment of 1.0. Such data might be rejected as outliers [3], or data from distances $>178^{\circ}$ could be systematically excluded.

The results include separate estimates of the station effects for both the horizontal and vertical components at 105 sites. This is a sufficiently large data-base to analyse for systematic differences between these two components. Overall, the station terms of the two components differ significantly at only five sites at the $1 \%$ level, and a further four sites at the $5 \%$ level. The former sites are "AFI" (Afiamalu, Samoa), "DBN" (De Bilt, The Netherlands), "RAR" (Rarotonga), "RIV" (Sydney) and "TAS" (Tashkent); the latter are "SBA" (Scott Base, Antarctica), "SHE" (Shanghai), "TFO" (Tonto Forest, Arizona), and "TUC" (Tucson, Arizona). For all of these sites except 
"SHE", the station term for the horizontal component exceeds that for the vertical component. It is notable that the nine sites listed include four with mean distances less than $40^{\circ}$, out of a total of only seven such sites in the data set. The differences at these four sites are supported by a high number of station observations and in three cases they are significant at the $1 \%$ level. In Figure 4, the difference between the horizontal and vertical station terms is plotted against mean distance from the earthquake sources. It can be seen that the difference tends to be positive at short distances, being about 0.2 at $20^{\circ}$ and decreasing to be about zero, on average, at distances greater than $90^{\circ}$, as shown by the robust smooth trend line. The positive tendency at distances less than $40^{\circ}$ is well supported by the data, as indicated above, but there are few stations to determine if there is any real difference in the $40^{\circ}-80^{\circ}$ range. A physical explanation for this tendency is lacking at present.

\section{CONCLUSION}

The station correction analysis presented here will facilitate the estimation of surface-wave magnitudes of future New Zealand earthquakes, as well as past events not included in the present data set [1]. The wide range of values found for the station terms underlines the importance of applying station corrections, especially when only a small number of observations is available for a given earthquake. The station terms cover a larger set of stations than have been included in a previous study, and generally have lower standard errors than previous estimates. The use of more stations will tend to increase the precision of magnitude estimates in future studies.

The relations between station term and distance and between absolute residual and distance have clarified the important role of stations at distances greater than $160^{\circ}$ in the estimation of surface-wave magnitudes of New Zealand earthquakes.

\section{ACKNOWLEDGEMENTS}

This study was funded by the Foundation for Research, Science and Technology under Contract No. C05506. We wish to thank J.X. Zhao and T.H. Webb for their constructive in-house reviews of this paper, and also an unknown reviewer.

\section{REFERENCES}

1. Dowrick, D.J. and Rhoades, D.A. (1998). Magnitudes of New Zealand earthquakes, 1901-1993, Bulletin N.Z. National Society for Earthquake Engineering, 31 (4), 260-280.

2. Dowrick, D.J. and Smith, E.G.C. (1990). Surface-wave magnitudes of some New Zealand earthquakes 19011988, Bulletin N.Z. National Society for Earthquake Engineering, 23 (3), 198-210.

3. Dowrick, D.J. (1991). Magnitude reassessment of New Zealand earthquakes, Earthquake Engineering and Structural Dynamics, 20, 577-596.

4. Bulletin of the International Seismological Centre (England), various issues.

5. Regional Catalogue of Earthquakes, International Seismological Centre (England), various issues.

6. Chambers, J.M. and Hastie T.J. (1992). Statistical Models in $S$, Wadsworth \& Brooks/Cole, Pacific Grove, California, 608pp.

7. Rial, J.A., and Cormier, V.F. (1980). Seismic waves at the epicenter's antipode, Journal of Geophysical Research, 85, B5, 2661-2668. 